\title{
Evaluation of the airway microbiome in nontuberculous mycobacteria disease
}

\author{
Imran Sulaiman (10), Benjamin G. Wu', Yonghua Li ${ }^{1}$, Adrienne S. Scott ${ }^{1}$, \\ Patrick Malecha ${ }^{1}$, Benjamin Scaglione ${ }^{1}$, Jing Wang ${ }^{1}{ }^{1}$, Ashwin Basavaraj ${ }^{1}$, \\ Samuel Chung ${ }^{1}$, Katrina Bantis ${ }^{1}$, Joseph Carpenito ${ }^{1}$, Jose C. Clemente ${ }^{3,4}$, \\ Nan Shen ${ }^{3}$, Jamie Bessich ${ }^{1}$, Samaan Rafeq ${ }^{1}$, Gaetene Michaud ${ }^{1}$, \\ Jessica Donington ${ }^{1}$, Charissa Naidoo ${ }^{5}$, Grant Theron ${ }^{5}$, Gail Schattner ${ }^{1}$, \\ Suzette Garofano ${ }^{1}$, Rany Condos ${ }^{1}$, David Kamelhar ${ }^{1}$, Doreen Addrizzo-Harris ${ }^{1}$ \\ and Leopoldo N. Segal ${ }^{1,2}$
}

Affiliations: ${ }^{1}$ Division of Pulmonary, Critical Care, and Sleep Medicine, New York University School of Medicine, New York, NY, USA. ${ }^{2}$ Dept of Medicine, New York University School of Medicine, New York, NY, USA. ${ }^{3}$ Dept of Genetics and Genomic Sciences and Immunology Institute, Icahn School of Medicine at Mount Sinai, New York, NY, USA. "Precision Immunology Institute, Icahn School of Medicine at Mount Sinai, New York, NY, USA. ${ }^{5}$ Medicine and Health Sciences, Stellenbosch University, DST/NRF of Excellence for Biomedical Tuberculosis Research and SA MRC Centre for Molecular and Cellular Biology, Division of Molecular Biology and Human Genetics Tygerberg, Cape Town, South Africa.

Correspondence: Leopoldo N. Segal, NYU School of Medicine, 462 First Ave 7W54, New York, NY 10016, USA. E-mail: leopoldo.segalanyumc.org

@ERSpublications

16S rRNA gene sequencing is not sensitive to detect Mycobacterium but identifies microbiota signatures associated with inflammation http://ow.ly/opXm30ldtQH

Cite this article as: Sulaiman I, Wu BG, Li Y, et al. Evaluation of the airway microbiome in nontuberculous mycobacteria disease. Eur Respir J 2018; 52: 1800810 [https://doi.org/10.1183/ 13993003.00810-2018].

ABSTRACT Aspiration is associated with nontuberculous mycobacterial (NTM) pulmonary disease and airway dysbiosis is associated with increased inflammation. We examined whether NTM disease was associated with a distinct airway microbiota and immune profile.

297 oral wash and induced sputum samples were collected from 106 participants with respiratory symptoms and imaging abnormalities compatible with NTM. Lower airway samples were obtained in 20 participants undergoing bronchoscopy. 16S rRNA gene and nested mycobacteriome sequencing approaches characterised microbiota composition. In addition, inflammatory profiles of lower airway samples were examined.

The prevalence of $\mathrm{NTM}^{+}$cultures was $58 \%$. Few changes were noted in microbiota characteristics or composition in oral wash and sputum samples among groups. Among $\mathrm{NTM}^{+}$samples, $27 \%$ of the lower airway samples were enriched with Mycobacterium. A mycobacteriome approach identified Mycobacterium in a greater percentage of samples, including some nonpathogenic strains. In $\mathrm{NTM}^{+}$lower airway samples, taxa identified as oral commensals were associated with increased inflammatory biomarkers.

The 16S rRNA gene sequencing approach is not sensitive in identifying NTM among airway samples that are culture-positive. However, associations between lower airway inflammation and microbiota signatures suggest a potential role for these microbes in the inflammatory process in NTM disease. 


\section{Introduction}

Nontuberculosis Mycobacterium (NTM) disease has an estimated incidence of 1.0-7.2 cases per 100000 persons per year, and its incidence is increasing for unknown reasons [1]. Despite widespread exposure to these organisms, only a minority of exposed individuals will acquire NTM, and an even smaller subgroup will develop clinically evident disease. Importantly, effectiveness of treatments for active NTM disease has been limited due to an absence of antimicrobial agents with low toxicity and good in vivo activity against the organism. Thus, treatment for NTM is not recommended for everyone, as the clinical course of the pulmonary disease is variable [2].

The currently clinically available methods of culturing airway samples are not able to represent interactions that NTM may have with other bacterial organisms present in a complex microbial environment. With improvements in culture-independent techniques, the microbiota-host immune interaction can be examined in further detail [3]. There have been several studies examining the airway microbiota in non-cystic fibrosis bronchiectasis [4-10] using sputum obtained from cohorts where NTM is not prevalent. However, description of the lower airway microbiome has been challenging because the bacterial burden in the lung is approximately a million-fold lower than in the gut and a hundred-fold lower than in the upper airway $[11,12]$. The presence of supraglottic microbes such as Veillonella or Prevotella in the lower airway is common [11-18], and they are associated with increased inflammation $[11,19]$, supporting the idea that microbiota changes are linked to the host immune phenotype in the airway mucosa. Therefore, we seek to identify microbial signatures associated with culture-positive NTM that may affect the host immune phenotype. Here, we utilised a $16 \mathrm{~S}$ rRNA gene high-throughput sequencing approach in parallel with a modified "mycobacteriome" sequencing approach on a large cohort of subjects with non-cystic fibrosis bronchiectasis and high prevalence of NTM.

\section{Material and methods}

\section{Study subjects}

This was a prospective observational study of non-HIV-infected patients $(n=106)$ with a diagnosis of non-cystic fibrosis bronchiectasis enrolled over a 2-year period at New York University (NYU) as part of a USA multicentre bronchiectasis cohort (Bronchiectasis Research Registry). All participants signed informed consent and the protocol was approved by the NYU institutional review boards (S14-01400). See the online supplementary methods for details on patient selection.

\section{Procedures}

Oral wash and induced sputum samples were collected from every patient at enrolment and again when sputum was clinically indicated over the 2 -year study period (online supplementary table S1). In addition, for every induced sputum we collected an oral wash sample prior to sputum induction. A portion of this sputum sample was sent to the clinical laboratory for culture and, based on the epithelial cell count, $90.5 \%$ of induced sputum samples met criteria for good quality [20]. Aliquots of oral wash and induced sputum were frozen at $-80^{\circ} \mathrm{C}$ for bacterial DNA sequencing. In order to investigate how reliable induced sputum was to evaluate the lower airway microbiota and to evaluate the inflammatory status of the lower airway mucosae, a subset of patients $(n=20)$ underwent bronchoscopy (clinically indicated in 14 cases, while the remaining six were undertaken for research-only purposes). Sampling included equipment background controls (sterile saline, Yankauer and bronchoscope), supraglottic (sampled using Yankauer) and two bronchoalveolar lavage (BAL) samples: one from an involved lung segment (predefined based on computed tomography (CT) scan) and the other from a non-involved lung segment. No samples were obtained from participants during an acute exacerbation or recent antibiotic use $(<1$ month). Whole $\mathrm{BAL}$ fluid aliquots were frozen at $-80^{\circ} \mathrm{C}$ for bacterial $16 \mathrm{~S}$ rRNA gene sequencing as well as $16 \mathrm{~S}$ quantitative (q)PCR.

Details of DNA sequencing are described in the online supplementary methods. In addition to 16S rRNA gene sequencing performed using Illumina MiSeq (San Diego, CA, USA), we used a nested PCR approach in parallel to enrich for Mycobacterium DNA coding for the 16S rRNA gene prior to library preparation for sequencing in order to describe the mycobacteriome, as previously published [21]. The obtained 16S rRNA gene sequences were analysed using the Quantitative Insights into Microbial Ecology (QIIME 1.9) package [22].

Immune profiling was performed for all BAL samples from the 20 patients who were part of the bronchoscopy subgroup. In vivo inflammation was assessed from acellular bronchoscopy samples by cell count differential and cytokines using Luminex 200 (Austin, TX, USA), as previously described [23, 24]. Ex vivo cytokine production of BAL cells was evaluated during toll-like receptor (TLR)4 stimulation (online supplementary methods). 


\section{Statistical analysis}

For association with discrete factors, we used nonparametric tests (Mann-Whitney or Kruskal-Wallis ANOVA). We used the ade4 package in $\mathrm{R}$ to construct principal coordinate analysis (PCoA) based on weighted UniFrac distances $[25,26]$. To cluster microbiome communities into exclusive "metacommunities" we used a Dirichlet multinomial mixture model with the $\mathrm{R}$ package DirichletMultinomial [27, 28]. To evaluate differences between groups of $16 \mathrm{~S}$ data or inferred metagenomes, we used linear discriminant analysis (LDA) effect size (LEfSe) [29]. For tests of association with continuous variables, we used nonparametric Spearman correlation tests. Co-occurrence between most abundant bacterial genera $(>1 \%$ relative abundance in at least one sample) were assessed using SparCC [30] with 20 iterations and 500 bootstrap replicates to eliminate correlations where significance was driven by outliers and visualised using Cytoscape v3.0.2 [31]. Only biomarkers that passed false discovery rate correction were used for this analysis [32]. All data are publicly available in the Sequence Read Archive (https://www.ncbi.nlm.nih.gov/sra) under accession number PRJNA418131. All codes used for the analysis included in this manuscript are available at https://github.com/segalmicrobiomelab/ntm_bronchiectasis_microbiome.

\section{Results}

\section{Participants}

Table 1 shows demographics and clinical characteristics of 106 patients. All participants had imaging abnormalities. Culture data show that 61 (58\%) out of 106 participants had positive NTM sputum cultures at baseline. Body mass index was lower among $\mathrm{NTM}^{+}$participants $(\mathrm{p}<0.01)$. Importantly, patients had variable prevalence of symptoms and radiographic findings.

\section{Comparing sputum and oral wash microbiome}

To evaluate the airway microbiome, we utilised all obtained oral wash and sputum samples ( $\mathrm{n}=297)$. Oral wash samples had higher $\alpha$-diversity than sputum (Shannon index; online supplementary figure S1a). Furthermore, $\beta$-diversity analysis showed significant differences between these two sample types (PERMANOVA $\mathrm{p}<0.001$; online supplementary figure S1b), although there was a greater degree of similarity between samples from the same subject than between subjects (online supplementary figure S1c). LEfSe analysis showed that sputum samples were enriched with Prevotella, Veillonella and Corynebacterium, while oral wash samples were enriched with Streptococcus, Rothia and Actinomyces (online supplementary figure S1d).

\section{Comparison of airway microbiota in $\mathrm{NTM}^{+}$versus $\mathrm{NTM}^{-}$using sputum and oral wash samples}

Next, we compared differences in microbiota for each of these sample types based on NTM status. The NTM culture status was based on the culture result of the specimen that was sequenced. Figure 1 and online supplementary figure S2 evaluate differences based on NTM culture status at the time of sample collection. In oral wash samples, there was no significant difference in $\alpha$-diversity, but a significant difference in $\beta$-diversity between $\mathrm{NTM}^{-}$and $\mathrm{NTM}^{+}$samples (PERMANOVA $\mathrm{p}=0.043$ ). In sputum samples, there were no statistically significant differences in either $\alpha$-diversity $(p=0.05)$ or $\beta$-diversity $(\mathrm{p}=0.08)$ between $\mathrm{NTM}^{-}$and $\mathrm{NTM}^{+}$samples. Similar negative results were seen when comparing NTM status based on American Thoracic Society/Infectious Diseases Society of America diagnostic criteria (NTM culture positivity in at least two sputum samples or one BAL sample) [33] or when only baseline sample were considered (data not shown).

Interestingly, Mycobacterium was not found to be enriched in $\mathrm{NTM}^{+}$samples. Indeed, this genus was only found present in a very small percentage of oral and sputum samples. Therefore, we investigated whether more differences in microbiota could be identified by sampling the lower airway.

\section{Microbiota comparison across the airways using bronchoscopic samples}

A subgroup of 20 participants from this cohort (40\% with culture-positive NTM) underwent bronchoscopy (online supplementary table S2 and figure S3). First, we compared how representative the sputum was of the lower airway microbiota (where we sampled involved and non-involved lung segments based on CT). Quantification of 16S rRNA copies using qPCR showed that sputum had approximately $\log _{2}$ higher bacterial load compared to BAL samples (online supplementary figure S4). The high bacterial load in sputum was comparable with the bacterial load present in oral wash and supraglottic samples. Figure 2 displays the $\alpha$ - and $\beta$-diversity for all bronchoscopy related samples. There were significant differences in $\beta$-diversity $(\mathrm{p}<0.01)$. The degree of similarity between upper airway, sputum and BAL samples was calculated as weighted UniFrac distance. Interestingly, sputum was more similar to oral wash or to supraglottic samples than to BAL (true for both involved or non-involved lung segments; figure 2c). These data suggest that sputum cannot be used as a surrogate for the lower airways to study the airway microbiota in this cohort. 
TABLE 1 Baseline demographic and clinical information of all patients recruited

\begin{tabular}{|c|c|c|c|c|}
\hline & \multirow[t]{2}{*}{ All patients } & \multicolumn{2}{|c|}{ NTM status } & \multirow[t]{2}{*}{$\mathrm{p}$-value } \\
\hline & & - & + & \\
\hline Subjects & 106 & 45 & 61 & \\
\hline Age years & $67.5 \pm 10.7$ & $67.6 \pm 10.1$ & $67.3 \pm 11.3$ & 0.88 \\
\hline Female & $95(89)$ & $37(82)$ & 58 (95) & 0.71 \\
\hline Caucasian & 92 (87) & $34(76)$ & 58 (95) & 0.39 \\
\hline BMI $\mathrm{kg} \cdot \mathrm{m}^{-2}$ & $22.7 \pm 4.3$ & $24.2 \pm 5.2$ & $21.5 \pm 3.1$ & $<0.01$ \\
\hline Smoking packs per day & $1.3 \pm 2.2$ & $1.2 \pm 1.0$ & $1.4 \pm 3.0$ & 0.85 \\
\hline Smoking years & $19.2 \pm 13.1$ & $22.9 \pm 14.5$ & $15.1 \pm 9.9$ & 0.04 \\
\hline \multicolumn{5}{|l|}{ Symptoms } \\
\hline Cough & $63(59)$ & $22(49)$ & $41(67)$ & 0.07 \\
\hline Sputum & $47(44)$ & $14(31)$ & $33(54)$ & 0.15 \\
\hline Haemoptysis & $8(8)$ & $3(7)$ & $5(8)$ & 0.76 \\
\hline Shortness of breath & 42 (39) & $17(38)$ & $25(41)$ & 0.92 \\
\hline Wheeze & $14(13)$ & $7(16)$ & $7(11)$ & 0.6 \\
\hline Fatigue & $26(25)$ & $9(20)$ & $17(28)$ & 0.6 \\
\hline Post-nasal drip & $36(34)$ & $19(42)$ & $17(28)$ & 0.03 \\
\hline Sinusitis & $4(4)$ & 4 [9] & $0(0)$ & 0.03 \\
\hline \multicolumn{5}{|l|}{ Current NTM culture } \\
\hline MAC & $56(53)$ & $0(0)$ & 56 (92) & $<0.001$ \\
\hline M. abscessus & $5(5)$ & $0(0)$ & $5(8)$ & $<0.05$ \\
\hline \multicolumn{5}{|l|}{ Current bacterial culture } \\
\hline Oropharyngeal flora & $15(14)$ & $4(9.5)$ & $11(18)$ & 0.13 \\
\hline$P$. aeruginosa & $6(6)$ & $1(2)$ & $5(8)$ & 0.19 \\
\hline MSSA & $5(5)$ & $2(4)$ & $3(5)$ & 0.91 \\
\hline Other & $10(9)$ & $4(9.5)$ & $6(10)$ & 0.89 \\
\hline Negative culture & $17(16)$ & $9(20)$ & $8(13)$ & 0.34 \\
\hline \multicolumn{5}{|l|}{ Lung physiology\# } \\
\hline FVC \% pred & $92.7 \pm 20.1$ & $92.6 \pm 20.4$ & $92.8 \pm 20.0$ & 0.97 \\
\hline FEV $1 \%$ pred & $83.7 \pm 21.8$ & $83.4 \pm 22.1$ & $84.0 \pm 21.8$ & 0.91 \\
\hline $\mathrm{FEV}_{1} / \mathrm{FVC} \%$ & $70.2 \pm 10.6$ & $69.2 \pm 10.7$ & $71.0 \pm 10.6$ & 0.44 \\
\hline RV \% pred & $121.8 \pm 31.7$ & $118.4 \pm 32.8$ & $124.5 \pm 31.0$ & 0.41 \\
\hline TLC \% pred & $106.4 \pm 18.5$ & $105.6 \pm 16.2$ & $106.9 \pm 20.2$ & 0.76 \\
\hline Dıco \% pred & $87.4 \pm 20.7$ & $90.7 \pm 23.7$ & $85.4 \pm 18.7$ & 0.35 \\
\hline \multicolumn{5}{|l|}{ HRCT thorax } \\
\hline Bronchiectasis & $79(75)$ & $34(76)$ & $45(78)$ & 0.45 \\
\hline Thickened airways & 52 (49) & $23(51)$ & $29(59)$ & 0.73 \\
\hline Mucoid impaction & $54(51)$ & $20(44)$ & $34(74)$ & 0.57 \\
\hline Nodules & $70(66)$ & $29(64)$ & $41(67)$ & 0.29 \\
\hline Tree-in-bud & $30(28)$ & $11(24)$ & $19(31)$ & 0.41 \\
\hline Ground-glass opacification & $31(29)$ & $17(38)$ & $14(23)$ & 0.16 \\
\hline \multicolumn{5}{|l|}{ Questionnaire data" } \\
\hline SGRQ total score & $27.5 \pm 19.3$ & $28.4 \pm 21.1$ & $26.8 \pm 18.1$ & 0.68 \\
\hline RSI total & $11.9 \pm 9.2$ & $13.7 \pm 9.4$ & $10.7 \pm 8.9$ & 0.12 \\
\hline FSSG total & $8.5 \pm 8.5$ & $9.8 \pm 9.7$ & $7.6 \pm 7.5$ & 0.22 \\
\hline EAT-10 total & $3.0 \pm 5.8$ & $3.0 \pm 5.6$ & $40.8 \pm 20.0$ & 0.97 \\
\hline
\end{tabular}

Data are presented as $\mathrm{n}$, mean \pm SD or $\mathrm{n}(\%)$, unless otherwise stated. NTM: nontuberculous mycobacteria; BMI: body mass index; MAC: Mycobacterium avium complex; M. abcessus: Mycobacterium abscessus: $P$. aeruginosa: Pseudomonas aeruginosa; MSSA: methicillin-sensitive Staphylococcus aureus; FVC: forced vital capacity; FEV1: forced expiratory volume in 1s; RV: residual volume; TLC: total lung capacity; $D L C O$ : diffusion capacity of the lung for carbon monoxide; HRCT: high-resolution computed tomography; SGRQ: St George's respiratory questionnaire; RSI: reflux symptom index; FSSG: frequency scale for the symptoms of gastro-oesophageal reflux disease; EAT-10: eating assessment tool. ${ }^{\#}: n=89 ;{ }^{\uparrow}: n=101$.

Comparison of airway microbiota in NTM+ versus $\mathrm{NTM}^{-}$using bronchoscopic samples

Of the patients that underwent bronchoscopy, 12 (60\%) out of 20 were $\mathrm{NTM}^{-}$and eight (40\%) out of 20 were $\mathrm{NTM}^{+}$. There were no significant differences in the bacterial load of $\mathrm{NTM}^{+}$versus $\mathrm{NTM}^{-}$lower airway samples ( $\mathrm{p}=$ nonsignificant). Online supplementary figure S5 shows no statistically significant differences in $\alpha$ - or $\beta$-diversity in BAL samples when categorised based on NTM culture status. Similarly, no differences were noted between BAL samples obtained as part of clinically indicated bronchoscopy as 


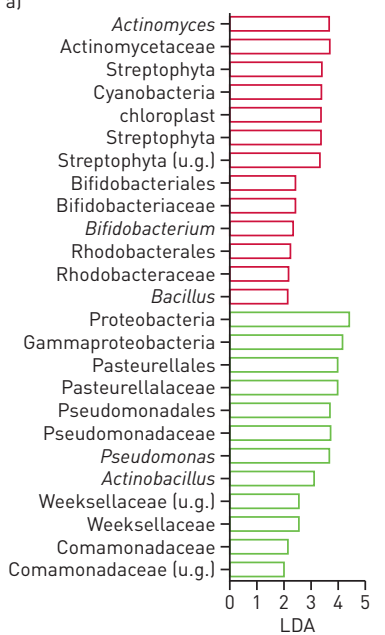

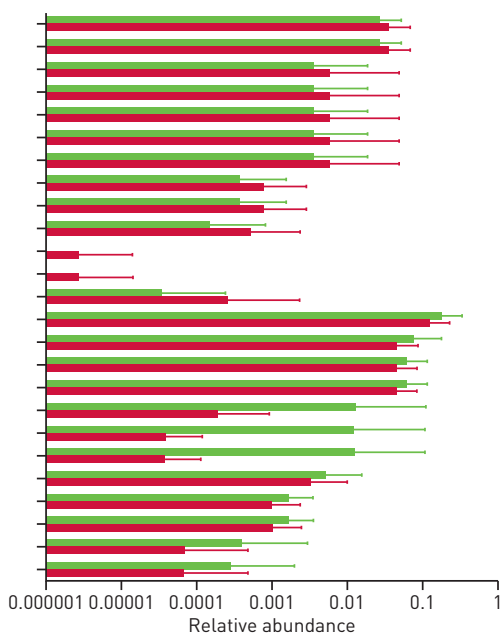
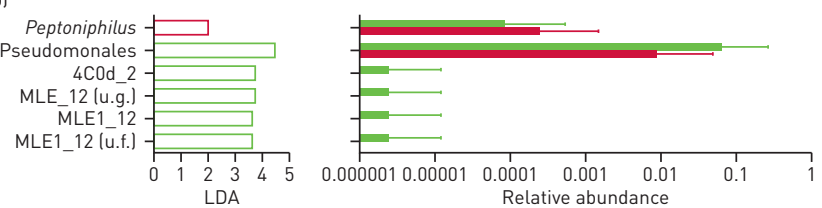

Enriched in:

$\square \mathrm{NTM}^{+}$

$\square$ NTM $^{-}$

FIGURE 1 Taxonomic differences between all oral wash and sputum samples for the nontuberculous mycobacteria (NTM) ${ }^{+}$and NTM $^{-}$groups. a) For oral wash samples, linear discriminant analysis (LDA) effect size (LEfSe) identified significant taxonomic differences in microbiome enrichment based on NTM status, but there was no enrichment with Mycobacterium in $\mathrm{NTM}^{+}$oral wash samples; b) for sputum, LEfSe detected few taxonomic differences and there was no enrichment with Mycobacterium in $\mathrm{NTM}^{+}$sputum samples. u.g.: undetermined genus; u.f.: undetermined family.

compared with research bronchoscopy (data not shown). Even though Mycobacterium was enriched in $\mathrm{NTM}^{+}$BAL samples (figure 3), this taxon was only present in $27 \%$ of these culture positive samples (median relative abundance $0(0-0.014)$ ). These data suggested that this sequencing approach was not able to detect the "pathogen" identified as responsible for the disease process and is consistent with prior literature that has shown poor accuracy of $16 \mathrm{~S}$ rRNA gene sequencing methods to detect Mycobacterium [21].
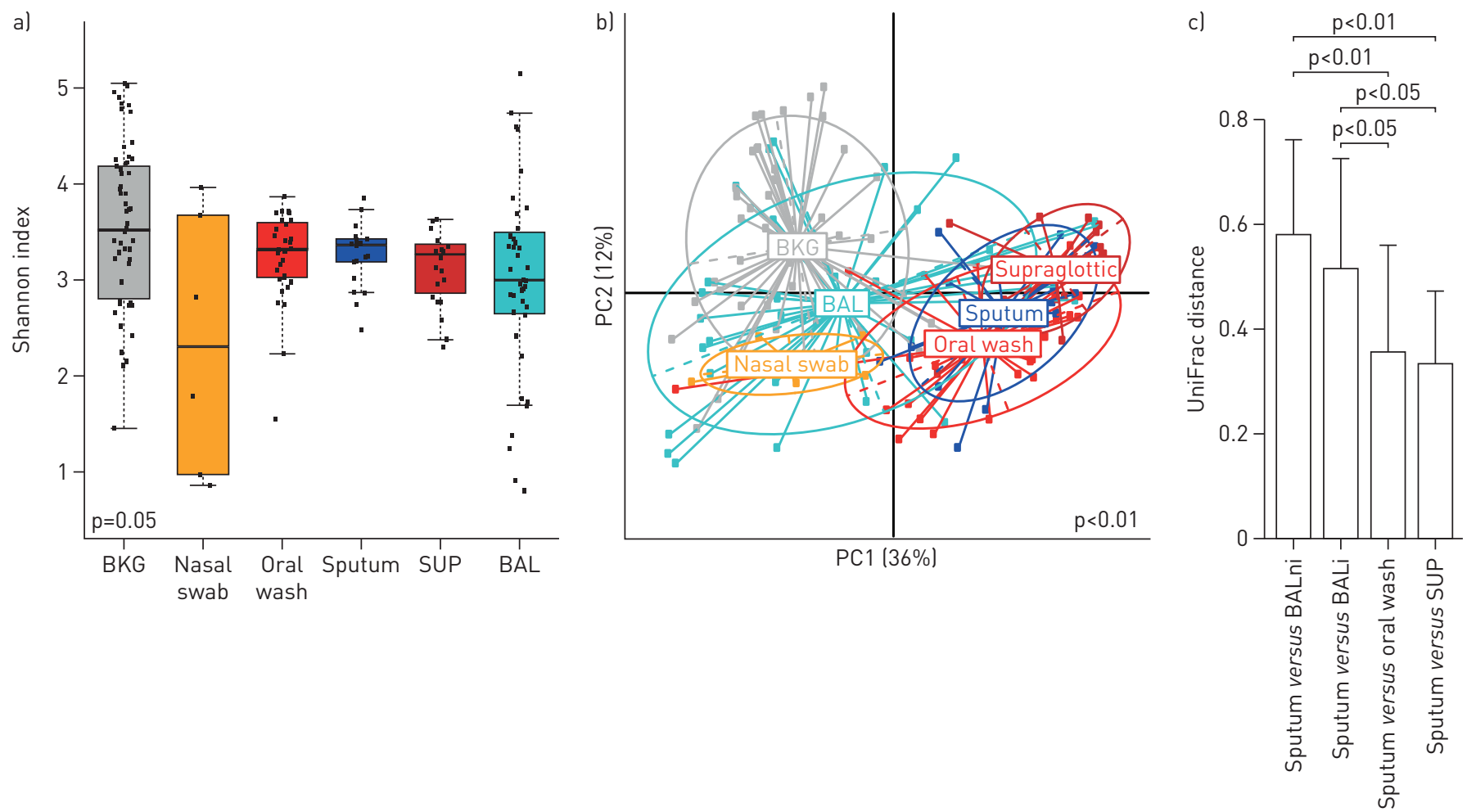

FIGURE 2 Evaluation of the lower airway microbiota using bronchoscopic samples. Bronchoscopy samples included background (BKG), nasal swab, oral wash, sputum, supraglottic (SUP) and bronchoalveolar lavage (BAL). a) There were significant differences in $\alpha$-diversity (Shannon diversity index) between all samples; b) $\beta$-diversity (based on weighted UniFrac) showed differential clustering based on sample type (PERMANOVA $\mathrm{p}$ <.01); c) comparison of the degrees of similarity between upper and lower airway samples based on UniFrac distance. Results showed that the microbiota in sputum were more similar to the microbiota of supraglottic and oral wash samples than to the microbiota in BAL samples (both involved (BALi) and non-involved (BALni)). 


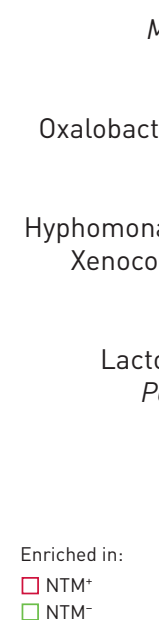

\section{Mycobacterium \\ Ralstonia \\ Clavibacter}

raceae (u.g.)

Enterobacter

Comamonas

phomonadaceae (u.g.)

Xenococcaceae (u.g.)

Paracoccus

3220 (u.g.)

Lactobillales (u.g.)

Porphyromonas

Mannheimia

Kaistobacter

Selenomonas

Dialister

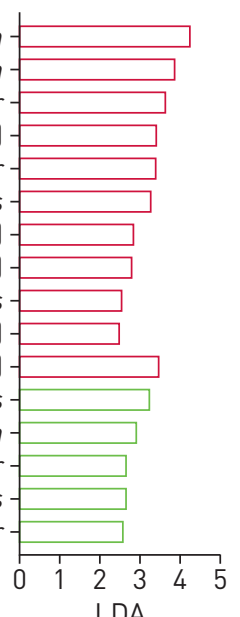

LDA

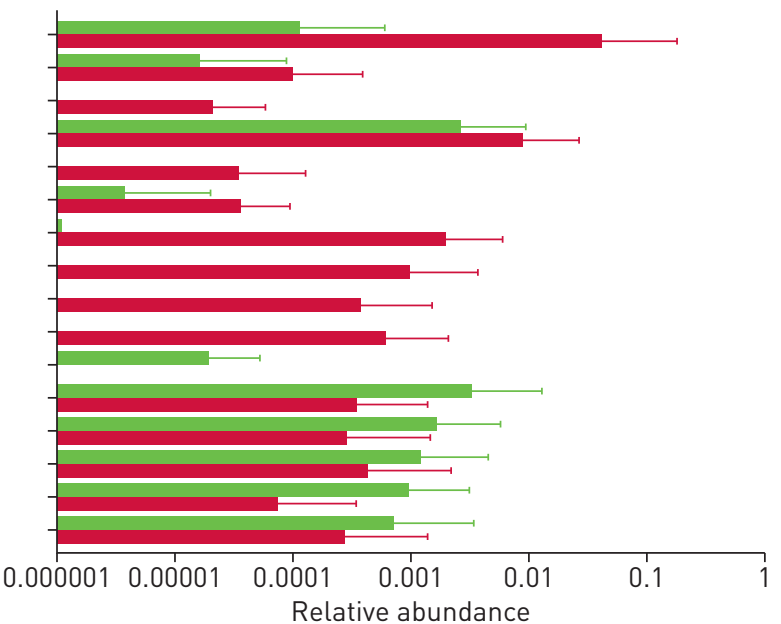

Relative abundance

FIGURE 3 Taxonomic differences between nontuberculous mycobacteria (NTM) ${ }^{+}$and $\mathrm{NTM}^{-}$in lower airway microbiota. Linear discriminant analysis (LDA) effect size analysis showed significant differences in lower airway microbiota composition enrichment based on NTM status. Unlike sputum samples, Mycobacterium was enriched in $\mathrm{NTM}^{+}$bronchoalveolar lavage samples.

\section{Evaluation of the mycobacteriome}

Therefore, we used an optimised protocol to enrich for Mycobacterium DNA coding for the 16S rRNA gene using a recently published DNA isolation method [34] and a nested PCR approach [35] (online supplementary material). First, we used a mock community of Mycobacteriun fortuitum and Streptococcus in order to establish the limit of detection for Mycobacterium with this approach (online supplementary results and online supplementary figure $\mathrm{S} 6$ for further details). We then used this nested mycobacteriome approach in all samples from the participants who underwent bronchoscopy and created PCoA plots comparing standard $16 \mathrm{~S}$ rRNA gene sequencing and nested mycobacteriome approaches of BAL, sputum and supraglottic samples. Figure 4 shows significant overlap for a large proportion of samples, but identifies compositional differences in others. In addition, histograms in figure 4 show the relative abundance of different Mycobacterium operational taxonomic units (OTUs) obtained with both nested mycobacteriome and 16S rRNA gene sequencing approaches. In BAL samples, Mycobacterium was detected in four (27\%) out of $15 \mathrm{NTM}^{+}$samples with standard 16S rRNA gene sequencing approaches, but with the nested mycobacteriome approach Mycobacterium was detected in all four samples plus three other $\mathrm{NTM}^{+}$BAL samples (47\%). Blast analysis demonstrate that these sequences matched Mycobacterium avium. Furthermore, this nested mycobacteriome approach identified one (5\%) out of $21 \mathrm{NTM}^{-}$samples with Mycobacterium. Blast analysis of this OTU was annotated to Mycobacterium houstonense (an environmental Mycobacterium not known to be pathogenic). Use of this nested mycobacteriome approach on background control samples detected a significant amount of Mycobacterium reads ( $>5 \%$ relative abundance) in only one control background sample, and fewer reads in only two out of 19 background equipment samples. In addition, blast analyses of Mycobacterium reads found in background equipment samples were annotated to a nonpathogenic strain (online supplementary figure S7). Similar results were found when this approach was utilised in oral and sputum samples from those subjects who did not undergo bronchoscopy (online supplementary material).

\section{Lower airway immunological profiling}

To evaluate the association of microbial signatures in NTM disease with a distinct mucosal immune phenotype we examined BAL cell differentials, in vivo cytokine levels and ex vivo cytokine production. In $\mathrm{NTM}^{+}$participants, BAL samples from involved lung segments had significantly higher neutrophils and fewer macrophages when compared to non-involved lung segments (table 2). In contrast, in $\mathrm{NTM}^{-}$ participants, BAL samples from involved lung segments had significantly higher lymphocytes. In vivo cytokine levels measured in BAL also showed a different inflammatory profile for $\mathrm{NTM}^{+}$. In $\mathrm{NTM}^{+}$ participants, BAL samples from involved lung segments had significantly higher levels of interferon (IFN)- $\gamma$, interleukin (IL)-8, IL-12p70, IFN- $\gamma$-inducible T-cell $\alpha$ chemoattractant (ITAC), macrophage inflammatory protein (MIP) $1 \alpha$ and MIP1B $\beta$, as compared with non-involved lung segments. This pattern was not present in BAL samples from $\mathrm{NTM}^{-}$participants, where involved lung segments had lower levels of MIP3 $\alpha$ and IL-17A. Similarly, a distinct inflammatory pattern was observed during ex vivo Toll-like receptor (TLR)-4 stimulation of BAL cells (online supplementary table S3). In $\mathrm{NTM}^{+}$participants, BAL 

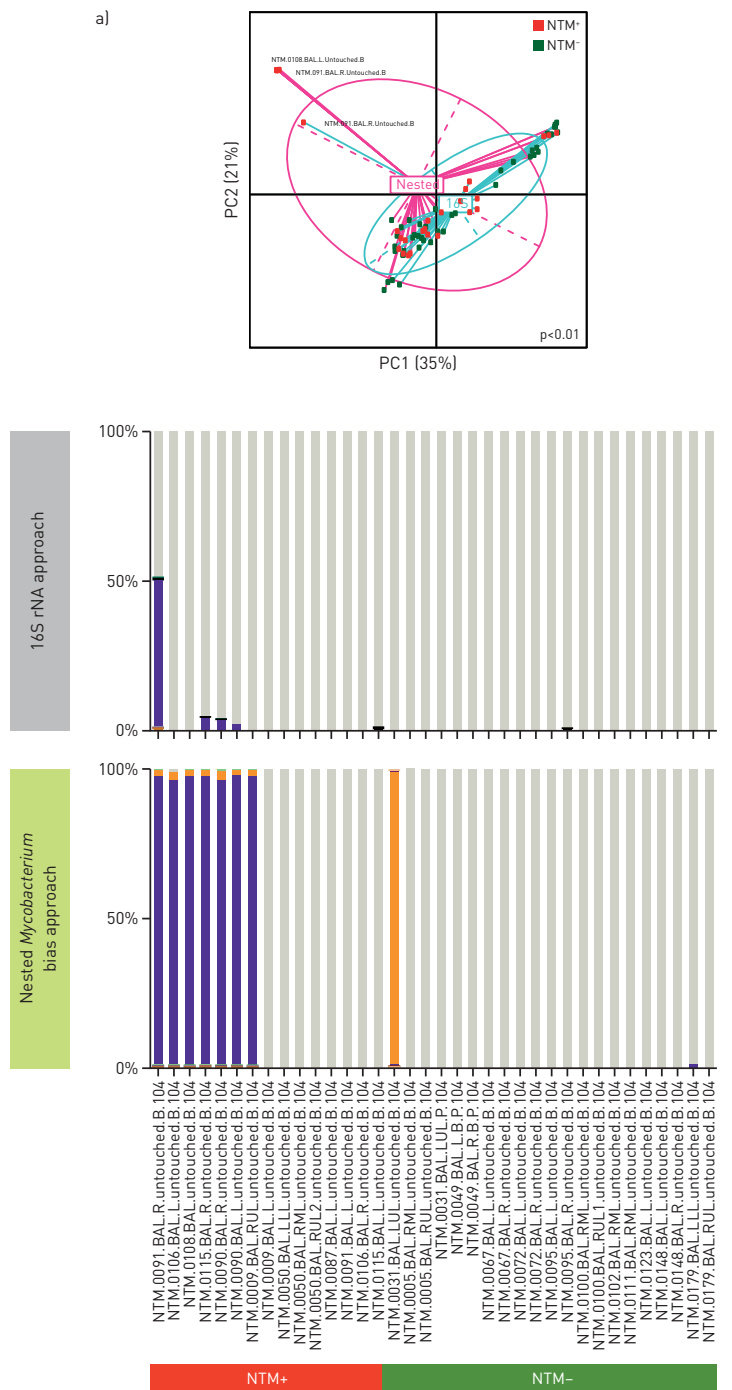
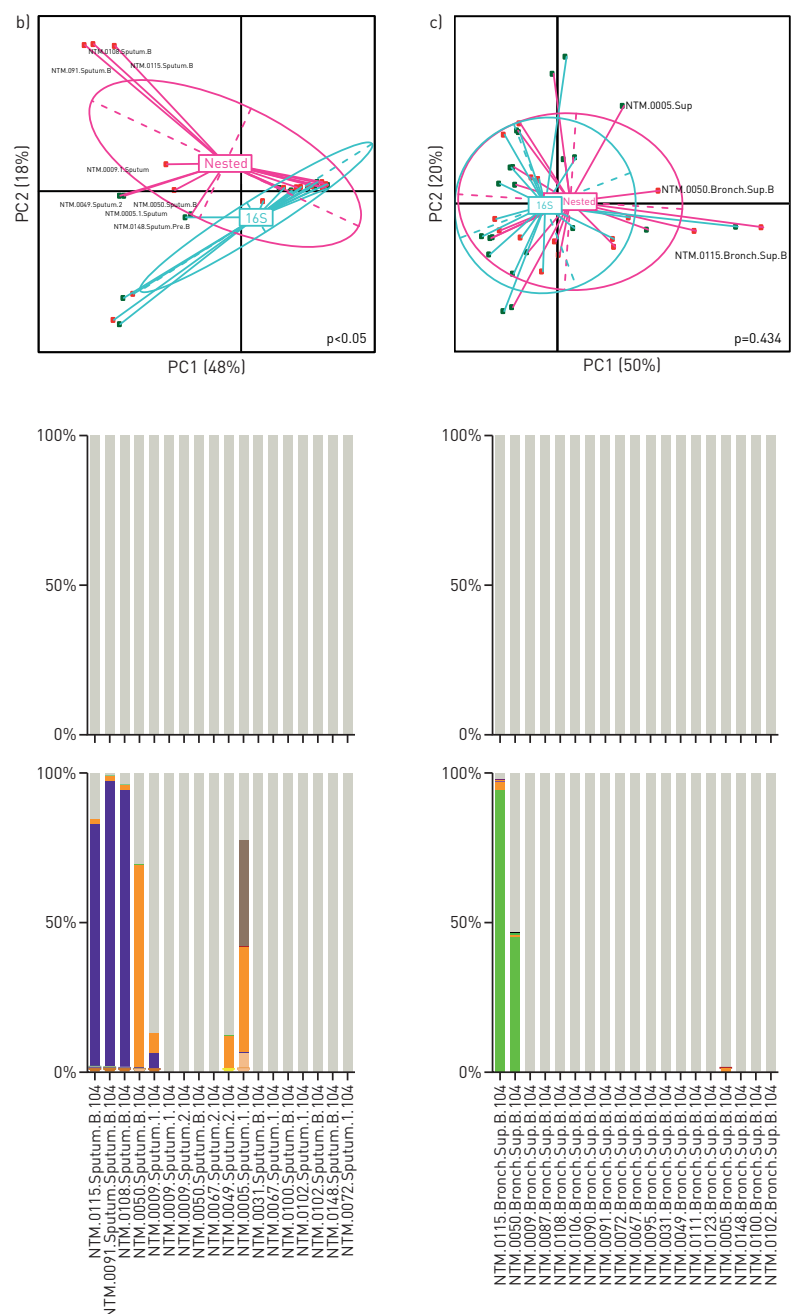

产
M. avium_ 978664 M. avium_4336253 M. avium_591521 M. houstonense 1062748 M. intermedium 514711 M. houstonense_546972 M. intermedium_13140 M. aurum_735466 M. insurbicum 215776 M. Moustonense 529762 Mintermedium 13069 M. intermedium_543570 M. septicum_711803 Non-Mycobacterium

FIGURE 4 Comparison between sequence data obtained using an unbiased $16 \mathrm{~S}$ rRNA approach and a biased mycobacteriome approach. Top panels show differences in $\beta$-diversity (based on weighted UniFrac distance) between samples processed with unbiased $16 \mathrm{~S}$ versus biased mycobacteriome approach. Bar charts in the bottom panels show relative abundance of operational taxonomic units (OTUs) annotated to Mycobacterium and their annotation based on BLAST on the two datasets. a) In bronchoalveolar lavage (BAL) there were significant differences in $\beta$-diversity between BAL samples processed with unbiased $16 \mathrm{~S}$ versus biased mycobacteriome approach (PERMANOVA p<0.01). With the biased mycobacteriome approach, Mycobacterium avium was identified in seven of the nontuberculous mycobacteria (NTM) ${ }^{+}$culture samples, while one $\mathrm{NTM}^{-}$sample had a Mycobacterium OTU annotated to $M$. houstonense (a strain not known to be pathogenic); b) in sputum there were significant differences in $\beta$-diversity between the sputum samples processed by unbiased $16 \mathrm{~S}$ versus biased mycobacteriome approach (PERMANOVA p<0.01). With the biased mycobacteriome approach, M. avium was identified in three of the $\mathrm{NTM}^{+}$culture samples; c) in supraglottic samples there was no significant difference in $\beta$-diversity between samples processed by unbiased $16 \mathrm{~S}$ versus biased mycobacteriome approach. In two samples, a Mycobacterium annotated to a nonpathogenic strain was identified.

cells from involved lung segments had blunted production of granulocyte-macrophage colony-stimulating factor (GM-CSF) and IFN- $\gamma$. These differences were not noted in $\mathrm{NTM}^{-}$participants.

Next, we evaluated the microbiome signatures associated with these inflammatory biomarkers in $\mathrm{NTM}^{+}$ samples and $\mathrm{NTM}^{-}$samples. For this, we used a network approach to evaluate taxa that trend to co-occur and were identified as distinct clusters based on Dirichlet multinomial model clustering (online supplementary material and figure S9).

In $\mathrm{BAL}$ of $\mathrm{NTM}^{+}$participants, oral commensals such as Prevotella, Veillonella and Leptotrichia tended to co-occur and had significant correlations with neutrophils and several cytokines including IL-6, IL-17, IL-23 and fractalkine (figure 5). Interestingly, Mycobacterium was in a separate co-occurrence cluster and had no significant correlation with inflammatory biomarkers. In BAL of $\mathrm{NTM}^{-}$samples, the relative abundance of oral commensals in the lower airway samples had fewer significant correlations with cytokines and BAL cells (online supplementary figure S10). 
TABLE 2 Cell count, and in vivo cytokine levels in bronchoalveolar lavage of 20 patients from the bronchoscopy cohort

\begin{tabular}{|c|c|c|c|c|c|c|}
\hline & \multicolumn{2}{|c|}{$\mathrm{NTM}^{-}$} & \multirow[t]{2}{*}{ p-value $\#$} & \multicolumn{2}{|c|}{$\mathrm{NTM}^{+}$} & \multirow[t]{2}{*}{$p$-value } \\
\hline & Involved & Non-involved & & Involved & Non-involved & \\
\hline Subjects $\mathrm{n}$ & \multicolumn{2}{|c|}{12} & \multicolumn{4}{|c|}{8} \\
\hline Macrophages & $44.2(28.2-75.7)$ & 75.4 (51.7-80.4) & NS & $19.6(15.75-39.65)$ & $75.4(66.0-85.0)$ & $0.02^{+}$ \\
\hline Neutrophils & $52.8(13.4-69.8)$ & $13.2(10.5-35.05)$ & NS & 79.6 (58.2-82.75) & $17.1(6.5-31.2)$ & $0.02^{+}$ \\
\hline Lymphocytes & $2.8(2.6-10.9)$ & $11.4(2.95-12.7)$ & 0.02 & $2.2(1.7-2.45)$ & $1.8(0.5-3.8)$ & NS \\
\hline ITAC & $19.39(19.4-224.4)^{\pi}$ & $224.4(135.6-274.9)$ & NS & $426.4(372.5-1135.8)^{\pi}$ & $469.73(352.8-489.7)$ & $0.02^{\S}$ \\
\hline MIP1 $\beta$ & $64.875(60.7-114.2)$ & 83.545 (61.8-109.2) & NS & 138.08 (134.1-188.8) & $111.83(71.8-141.4)$ & $0.02^{\S}$ \\
\hline $\mathrm{IFN}-\gamma$ & $21.36(6.4-30)$ & $8.46(6.7-13.8)$ & NS & $63.085(24.8-158.6)$ & $22.095(17.6-26.5)$ & $0.03^{\S}$ \\
\hline IL-8 & $423.565(216.9-486.8)^{\pi}$ & $351.12(240.7-1810.2)$ & NS & $5927.82(5927.8-5927.8)^{\pi}$ & $411.345(242.8-586.8)$ & $0.03^{\S}$ \\
\hline IL-12 p70 & $3.95(3.7-4.1)^{9}$ & $3.715(3.5-4.3)$ & NS & $5.155(4.2-6.6)^{9}$ & $4.605(4.3-4.6)$ & 0.06 \\
\hline MIP1 $\alpha$ & $116.88(66.2-179.7)$ & 95.855 (72.4-128.3) & NS & $268.83(143.4-489.8)$ & $117.975(78.5-143.1)$ & 0.06 \\
\hline Fractalkine & $364.12(364.1-391.3)^{\pi}$ & $391.255(360.4-449.6)$ & NS & $488.115(455.5-544.1)^{9}$ & 558.94 (403.9-558.9) & NS \\
\hline $\mathrm{IL}-1 \beta$ & $22.27(1.9-11.1)$ & $3.94(2-6.5)$ & NS & $34.345(3.8-72.4)$ & $2.405(2.1-65.3)$ & NS \\
\hline IL-6 & $11.275(10.1-104)^{\pi}$ & $104.025(58.9-154.3)$ & NS & $82.735(60.9-133.4)^{\text {ก }}$ & 114.99 (69.7-147.3) & NS \\
\hline IL-21 & $4.825(4.5-6.5)$ & $5.95(4.7-7.3)$ & NS & $6.655(6-8.8)$ & 7.45 (5.4-9.17) & NS \\
\hline IL-7 & $15.935(15.2-38.4)$ & $24.97(16.1-36.4)$ & NS & $27.365(26.7-31.6)$ & $29.545(22.8-33.6)$ & NS \\
\hline IL-5 & $32.475(13.4-40.3)$ & $17.03(12.1-23.2)$ & NS & $26.395(23.9-31.4)$ & $56.18(56.2-18.4)$ & NS \\
\hline $\mathrm{IL}-23$ & $158.025(88.4-160.6)$ & $118.66(88.4-149.9)$ & NS & 153.865 (126.4-173.7) & $252.9(165.4-359.9)$ & NS \\
\hline TNF- $\alpha$ & $208.485(36.1-231.5)$ & 44.63 (37.5-105.5) & NS & 90.83 (67.3-130.3) & 54.845 (39.4-105.2) & NS \\
\hline GM-CSF & $19.225(18.1-41.2)$ & 29.935 (19.6-40) & NS & $35.795(32.1-42.2)$ & $27.215(22.5-37.0)$ & NS \\
\hline IL-4 & $29.09(22-30.5)$ & $25.42(22.9-32.6)$ & NS & $39.855(31-50.2)$ & $46.31(37.1-49.0)$ & NS \\
\hline IL-10 & $61.015(50.8-84.6)$ & $66.315(55.1-74)$ & NS & $45.74(32.1-68.6)$ & $83.5(68.2-89.1)$ & NS \\
\hline
\end{tabular}

\section{Discussion}

The purpose of this investigation was to evaluate the airway microbiota using culture-independent techniques in a prospective cohort of patients suspected of having NTM disease. Our analysis showed that using sputum samples, few changes in microbiota composition could be identified between samples with and without NTM identified by culture. Using upper and lower airway samples from a subgroup of participants who underwent bronchoscopy we showed that induced sputum offers a poor representation of the lower airway microbiota in this patient population and more accurately reflects the composition of the oral cavity. Furthermore, the culture-independent approach did not find Mycobacterium in a large percentage of samples. We expanded these observations with a Mycobacterium-biased nested sequence approach to confirm that in the majority of $\mathrm{NTM}^{+}$participants the abundance for this organism was low or not detected in contrast with the many other microbes identified. These data demonstrate the limited sensitivity of these culture-independent methods to detect Mycobacterium and exemplify an unrecognised limitation of current universal sequencing methods to study pathogens present at low abundance. Finally, the lower airways of $\mathrm{NTM}^{+}$participants had a distinct immunological phenotype, in which levels of several inflammatory biomarkers correlated with the relative abundance of microbes identified as oral taxa and not with the relative abundance of Mycobacterium. These data suggest that micro-aspiration and/or failure to clear aspirated oral microbes may contribute to the inflammatory endotype in NTM disease.

Culture-independent techniques have demonstrated that the airways harbour a complex microbiota that has a significant impact on the host immune response [3, 11, 23, 36, 37]. In a recent study involving 76 non-cystic fibrosis bronchiectasis patients from a multicentre European cohort, Haemophilus influenzae, Pseudomonas aeruginosa and Streptococcus were found to be the most abundant species in sputum samples [4]. However, this cohort is characterised by a low prevalence of NTM. In the United States, 


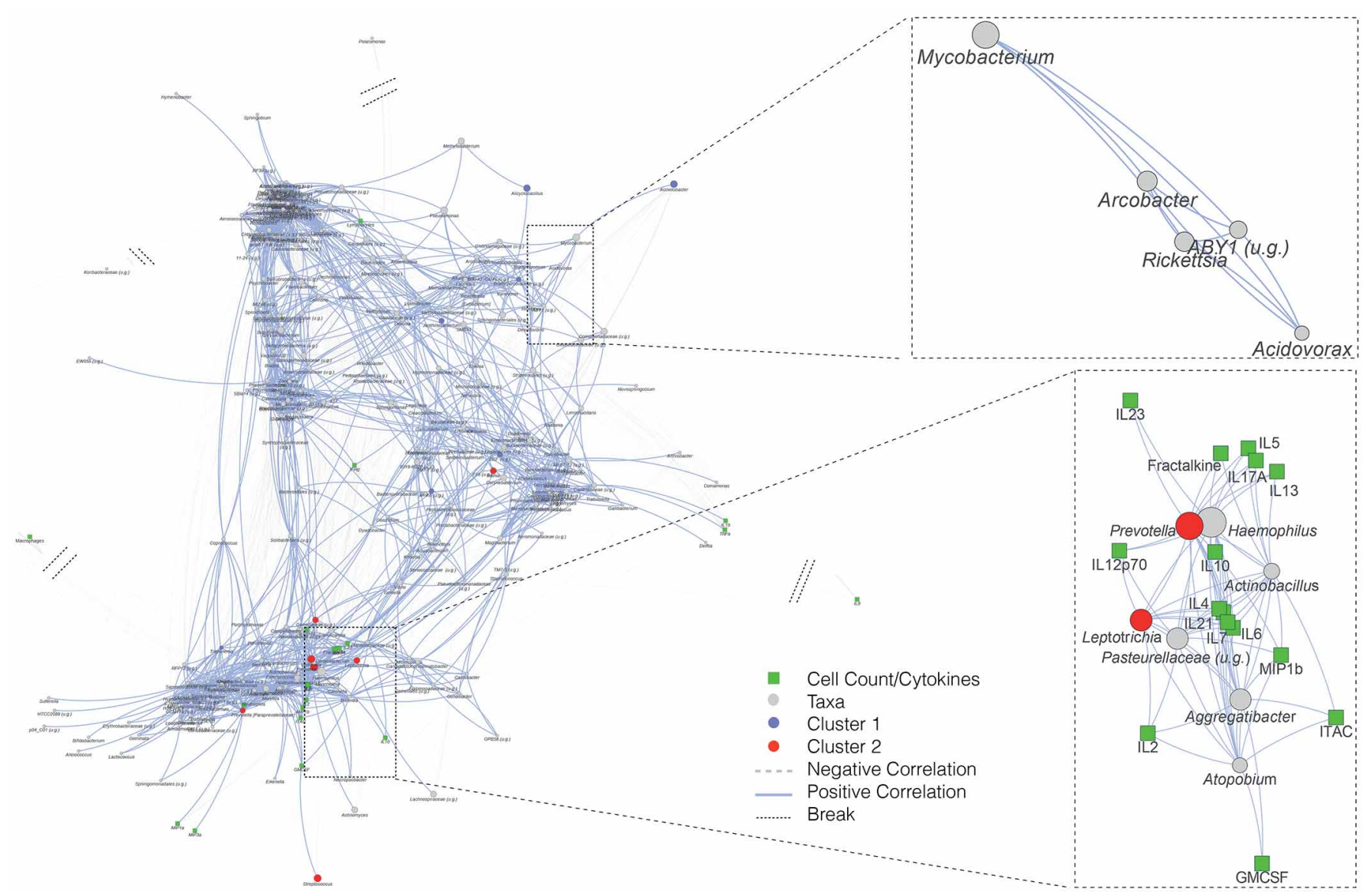

FIGURE 5 Associations between taxa and inflammatory biomarkers in the lower airways for nontuberculous mycobacteria (NTM) ${ }^{+}$samples. A network analysis using SparCC was constructed, to remove compositional and sparsity effects common in microbiome data, in order to identify correlations between taxa at a genus level and both inflammatory biomarkers. Each node represents a genus, with the size of nodes indicating the log-relative abundance from large (high) to small (low). In addition, taxa identified as markers for lower airway microbiota clusters (based on DMM analysis shown in online supplementary figure S5) were colour coded. Edges between nodes represent significant correlations, with the length of the edge representing the correlation coefficient strength (shorter edges indicating higher positive correlation). u.g.: undetermined genus.

NTM is a frequent cause of non-cystic fibrosis bronchiectasis, as recently shown by АкsАмiт et al. [38] and multiple strains of NTM are associated with bronchiectasis [39]. In our study, the prevalence of NTM was $58 \%$, similar to the US Bronchiectasis Research Registry [38]. Diagnosis of NTM disease is commonly based on induced sputum. Therefore, we examined microbiota differences in induced sputum as well as oral wash in our cohort. Both sputum and oral wash samples showed nonsignificant differences in diversity metrics based on NTM status. Moreover, in $\mathrm{NTM}^{+}$patients, Mycobacterium was not enriched in these samples.

To further characterise the lower airway microbiota in NTM disease a subset of patients underwent bronchoscopy with BAL and upper airway (oropharyngeal) sampling. Differences among oropharyngeal microbiota included enrichment with Streptococcus and Rothia in oral wash and enrichment with Prevotella and Veillonella in supraglottic samples. Importantly, comparison between induced sputum and both upper and lower airway samples showed that induced sputum is compositionally more similar to the upper airway microbiota (either oral wash or supraglottic) than to the lower airway microbiota. This supports that induced sputum is predominantly influenced by the composition of the upper airway microbiota and offers a poor representation of the lower airway microbiota. Similar to our findings, the use of induced sputum to evaluate the airway microbiota in a cohort of patients with asthma provided an incomplete reflection of the lower airways, mostly influenced by the oral microbiota [40]. This has implications for our limited understanding of the lower airway microbiota using non-invasive samples. In our cohort, BAL samples from $\mathrm{NTM}^{+}$participants were enriched with Mycobacterium and Oxalobacteraceae, while BAL samples from $\mathrm{NTM}^{-}$participants were enriched with Porphyromonas. However, similar to induced sputum, Mycobacterium was frequently not identified using 16S rRNA gene sequencing in samples with positive cultures for this organism. This is consistent with prior published 
observations [21, 34]. As NTM tends to have only one or two 16S rRNA genes per genome, they can be underrepresented in the context of other taxa with more 16S rRNA genes per bacteria using standard methods of $16 \mathrm{~S}$ sequencing $[33,41]$. In BAL, Mycobacterium was identified only in $27 \%$ of the $\mathrm{NTM}^{+}$ samples using standard 16S rRNA gene sequencing. For this investigation we seek to use a universal sequencing approach that allows us to characterise broadly the bacterial composition of the airway microbiota. Since Mycobacterium was rarely present among $\mathrm{NTM}^{+}$cases using the now broadly accepted $16 \mathrm{~S}$ rRNA gene sequencing, we attempted to improve our sensitivity in identifying this genus by applying a nested amplification approach where the first PCR targeted a Mycobacterium-specific region that contains the V4 region of the 16S rRNA gene [21, 42-44]. It is possible that other Mycobacterium-specific primers would have a better yield at identifying this organism. Nonetheless, with this method of sequencing, we were able to identify Mycobacterium in $47 \%$ of the BAL samples where NTM grew in culture. In contrast, Mycobacterium was only identified in $17 \%$ of $\mathrm{NTM}^{+}$sputum samples, which is probably also related to how representative the sputum is of the lower airway microbiota.

Our bronchoscopic sampling also allowed us to compare the inflammatory phenotype of $\mathrm{NTM}^{+}$and $\mathrm{NTM}^{-}$participants. It has previously been shown that with mycobacterial infection, through induction of IFN- $\gamma$, activated macrophages upregulate the expression of pro-inflammatory cytokines to help protect against mycobacterial infection. These cytokines include IL-6, IL-1 $\beta$, IL-12, tumour necrosis factor- $\alpha$ and nitric oxide $[45,46]$. In $\mathrm{NTM}^{+}$BAL samples obtained from involved sites, BAL cells stimulated with lippolysaccharide (LPS) showed significantly blunted IFN- $\gamma$ and GM-CSF levels, suggesting important impaired innate immune responses. In a co-occurrence network analysis, significant associations between taxa identified as oral commensals (e.g. Prevotella, Veillonella and Leptotrichia) and T-helper-17 cytokines were also seen in $\mathrm{NTM}^{+}$BAL samples. The relative abundance of Mycobacterium was not significantly correlated with levels of inflammatory biomarkers suggesting the importance of other microbes on the lower airway inflammatory tone in NTM disease.

There were several limitations in this study. The patients enrolled in this cohort had a mild form of NTM. A large proportion of patients with NTM grew Mycobacterium avium complex, consistent with the US Bronchiectasis Research Registry [33,38]. It is possible that different strains of Mycobacterium and different degrees of disease severity would have different airway microbiota and inflammatory signatures than that observed in our study. In this investigation, a relatively small proportion of patients underwent bronchoscopy allowing us to evaluate the lower airways [47] and most of these investigations were undertaken for clinical reasons. Although similar to our larger cohort, these patients may represent a different disease phenotype. However, given the limited representation of the lower airways provided by the microbiota present in sputum samples, further investigation is warranted in a larger cohort to uncover microbiota host interactions that might be relevant in this disease and to define which microbiota signatures present in induced sputum could be used to explore the lower airway microbiota. Furthermore, even though we identified some significant associations between lower airway microbiota signatures and inflammatory biomarkers in the $\mathrm{NTM}^{+}$group, we considered these results as exploratory and hypothesis-generating. A larger cohort of patients where bronchoscopic samples are obtained will be needed to confirm and expand these findings. Finally, the purpose of this study was to evaluate the microbiome community of patients with NTM-related bronchiectasis. Therefore, we did not evaluate the change in microbiome composition with exacerbations nor the effects of treatment for NTM. Importantly, patients on treatment for NTM were excluded from this analysis to avoid this potential confounder to the analysis of differences between $\mathrm{NTM}^{+}$versus $\mathrm{NTM}^{-}$groups.

In summary, we identified the limitations of current unbiased culture-independent techniques to identify Mycobacterium in patients with culture positivity for NTM, which highlights the need for technical improvements in these methods. In addition, we describe how patients with NTM disease have a distinct inflammatory environment in the lower airways that may be associated with some of the components of the lower airway microbiota including taxa commonly identified as oral commensals. These data suggest a possible role of micro-aspiration or failure to clear upper airway microbes from the lower airways and may explain some of the heterogeneity in presentation and disease progression among participants with culture-positive NTM disease. The contribution of the lower airway microbiota to the pathophysiological inflammatory process in NTM disease warrants further investigation in a larger cohort and may have potential therapeutic implications.

Author contributions: All authors listed contributed to this manuscript. I. Sulaiman and L.N. Segal were involved in conception and design. B. Scaglione, J. Wang, A. Basavaraj, Y. Li, A.S. Scott, S. Chung, K. Bantis, J. Bessich, J. Carpenito, S. Rafeq, G. Michaud, J. Donington, G. Schattner, S. Garofano, R. Condos, D. Kamelhar, D. Addrizzo-Harris, I. Sulaiman and L.N. Segal were all involved in acquisition of data. B.G. Wu, P. Malecha, J.C. Clemente, N. Shen, C. Naidoo, G. Theron, I. Sulaiman and L.N. Segal were involved in analysis and interpretation 
of data. B.G. Wu, Y. Li, J. Wang, P. Malecha, J.C. Clemente, N. Shen, G. Theron, J. Bessich, K. Bantis, S. Chung, S. Rafeq, G. Michaud, J. Donington, R. Condos, C. Naidoo, A. Basavaraj, A.S. Scott, D. Kamelhar, D. Addrizzo-Harris, I. Sulaiman and L.N. Segal were involved in drafting and revising of this manuscript. I. Sulaiman and L.N. Segal were involved in the final approval of this manuscript.

Support statement: This study was funded by the National Institutes of Health K23 AI102970 (to L.N. Segal), National Institutes of Health T32 CA193111 (to B.G. Wu), Helaine Lerner Fund (to L.N. Segal), NTM Info and Research (to L.N. Segal), Flight Attendant Medical Research Institute Young Clinical Scientist Award (to B.G. Wu), and Stony Wold Herbert Foundation Fellowship (to B.G. Wu). Funding information for this article has been deposited with the Crossref Funder Registry.

Conflict of interest: None declared.

\section{References}

1 Cassidy PM, Hedberg K, Saulson A, et al. Nontuberculous mycobacterial disease prevalence and risk factors: a changing epidemiology. Clin Infect Dis 2009; 49: e124-e129.

2 Saleeb P, Olivier KN. Pulmonary nontuberculous mycobacterial disease: new insights into risk factors for susceptibility, epidemiology, and approaches to management in immunocompetent and immunocompromised patients. Curr Infect Dis Rep 2010; 12: 198-203.

3 Segal LN, Rom WN, Weiden MD. Lung microbiome for clinicians. New discoveries about bugs in healthy and diseased lungs. Ann Am Thorac Soc 2014; 11: 108-116.

4 Cox MJ, Turek EM, Hennessy C, et al. Longitudinal assessment of sputum microbiome by sequencing of the 16S rRNA gene in non-cystic fibrosis bronchiectasis patients. PLoS One 2017; 12: e0170622.

5 Tunney MM, Einarsson GG, Wei L, et al. Lung microbiota and bacterial abundance in patients with bronchiectasis when clinically stable and during exacerbation. Am J Respir Crit Care Med 2013; 187: 1118-1126.

6 Boyton RJ, Reynolds CJ, Quigley KJ, et al. Immune mechanisms and the impact of the disrupted lung microbiome in chronic bacterial lung infection and bronchiectasis. Clin Exp Immunol 2013; 171: 117-123.

7 Rogers GB, van der Gast CJ, Cuthbertson L, et al. Clinical measures of disease in adult non-CF bronchiectasis correlate with airway microbiota composition. Thorax 2013; 68: 731-737.

8 Rogers GB, Zain NM, Bruce KD, et al. A novel microbiota stratification system predicts future exacerbations in bronchiectasis. Ann Am Thorac Soc 2014; 11: 496-503.

9 Maughan H, Cunningham KS, Wang PW, et al. Pulmonary bacterial communities in surgically resected noncystic fibrosis bronchiectasis lungs are similar to those in cystic fibrosis. Pulm Med 2012; 2012: 746358.

10 Purcell P, Jary H, Perry A, et al. Polymicrobial airway bacterial communities in adult bronchiectasis patients. BMC Microbiol 2014; 14: 130.

11 Segal LN, Alekseyenko AV, Clemente JC, et al. Enrichment of lung microbiome with supraglottic taxa is associated with increased pulmonary inflammation. Microbiome 2013; 1: 19.

12 Bassis CM, Erb-Downward JR, Dickson RP, et al. Analysis of the upper respiratory tract microbiotas as the source of the lung and gastric microbiotas in healthy individuals. MBio 2015; 6: e00037.

13 Charlson ES, Bittinger K, Haas AR, et al. Topographical continuity of bacterial populations in the healthy human respiratory tract. Am J Respir Crit Care Med 2011; 184: 957-963.

14 Erb-Downward JR, Thompson DL, Han MK, et al. Analysis of the lung microbiome in the "healthy" smoker and in COPD. PLoS One 2011; 6: e16384.

15 Morris A, Beck JM, Schloss PD, et al. Comparison of the respiratory microbiome in healthy non-smokers and smokers. Am J Respir Crit Care Med 2013; 187: 1067-1075.

16 Pragman AA, Kim HB, Reilly CS, et al. The lung microbiome in moderate and severe chronic obstructive pulmonary disease. PLoS One 2012; 7: e47305.

17 Sze MA, Dimitriu PA, Hayashi S, et al. The lung tissue microbiome in chronic obstructive pulmonary disease. Am J Respir Crit Care Med 2012; 185: 1073-1080.

18 Lozupone C, Cota-Gomez A, Palmer BE, et al. Widespread colonization of the lung by Tropheryma whipplei in HIV infection. Am J Respir Crit Care Med 2013; 187: 1110-1117.

19 Dickson RP, Erb-Downward JR, Prescott HC, et al. Cell-associated bacteria in the human lung microbiome. Microbiome 2014; 2: 28.

20 Weiszhar Z, Horvath I. Induced sputum analysis: step by step. Breathe 2013; 9: 300-306.

21 Macovei L, McCafferty J, Chen T, et al. The hidden "mycobacteriome" of the human healthy oral cavity and upper respiratory tract. J Oral Microbiol 2015; 7: 26094.

22 Caporaso JG, Kuczynski J, Stombaugh J, et al. QIIME allows analysis of high-throughput community sequencing data. Nat Methods 2010; 7: 335-336.

23 Segal LN, Clemente JC, Tsay JC, et al. Enrichment of the lung microbiome with oral taxa is associated with lung inflammation of a Th17 phenotype. Nat Microbiol 2016; 1: 16031.

24 Berger KI, Pradhan DR, Goldring RM, et al. Distal airway dysfunction identifies pulmonary inflammation in asymptomatic smokers. ERJ Open Res 2016; 2: 00066-2016.

25 Dray S, Dufour AB. The ade4 package: implementing the duality diagram for ecologists. J Stat Softw 2007; 22: $1-20$.

26 Lozupone $\mathrm{C}$, Lladser ME, Knights $\mathrm{D}$, et al. UniFrac: an effective distance metric for microbial community comparison. ISME J 2011; 5: 169-172.

27 Holmes I, Harris K, Quince C. Dirichlet multinomial mixtures: generative models for microbial metagenomics. PLoS One 2012; 7: e30126.

28 Morgan M. DirichletMultinomial: Dirichlet-Multinomial Mixture Model Machine Learning for Microbiome Data. $\mathrm{R}$ package version 1.20 .0 ed2017.

29 Segata N, Izard J, Waldron L, et al. Metagenomic biomarker discovery and explanation. Genome Biol 2011; 12: R60. 
Friedman J, Alm EJ. Inferring correlation networks from genomic survey data. PLoS Comput Biol 2012; 8: e1002687.

31 Shannon P, Markiel A, Ozier O, et al. Cytoscape: a software environment for integrated models of biomolecular interaction networks. Genome Res 2003; 13: 2498-2504.

32 Reiner A, Yekutieli D, Benjamini Y. Identifying differentially expressed genes using false discovery rate controlling procedures. Bioinformatics 2003; 19(3): 368-375.

33 Griffith DE, Aksamit T, Brown-Elliott BA, et al. An official ATS/IDSA statement: diagnosis, treatment, and prevention of nontuberculous mycobacterial diseases. Am J Respir Crit Care Med 2007; 175: 367-416.

34 Caverly LJ, Carmody LA, Haig SJ, et al. Culture-independent identification of nontuberculous mycobacteria in cystic fibrosis respiratory samples. PLoS One 2016; 11: e0153876.

35 Kauppinen J, Mäntyjärvi R, Katila ML. Mycobacterium malmoense-specific nested PCR based on a conserved sequence detected in random amplified polymorphic DNA fingerprints. J Clin Microbiol 1999; 37: 1454-1458.

36 Segal LN, Clemente JC, Wu BG, et al. Randomised, double-blind, placebo-controlled trial with azithromycin selects for anti-inflammatory microbial metabolites in the emphysematous lung. Thorax 2017; 72: 13-22.

37 Shenoy MK, Iwai S, Lin DL, et al. Immune response and mortality risk relate to distinct lung microbiomes in patients with HIV and pneumonia. Am J Respir Crit Care Med 2017; 195: 104-114.

38 Aksamit TR, O'Donnell AE, Barker A, et al. Adult patients with bronchiectasis: a first look at the US bronchiectasis research registry. Chest 2017; 151: 982-992.

39 Iseman MD, Marras TK. The importance of nontuberculous mycobacterial lung disease. Am J Respir Crit Care Med 2008; 178: 999-1000.

40 Durack J, Huang YJ, Nariya S, et al. Bacterial biogeography of adult airways in atopic asthma. Microbiome 2018; 6: 104 .

41 Danielides V, Patrikakos G, Moerman M, et al. Diagnosis, management and surgical treatment of non-tuberculous mycobacterial head and neck infection in children. ORL J Otorhinolaryngol Relat Spec 2002; 64: 284-289.

42 Kim BJ, Lee SH, Lyu MA, et al. Identification of mycobacterial species by comparative sequence analysis of the RNA polymerase gene (rpoB). J Clin Microbiol 1999; 37: 1714-1720.

43 Yamada-Noda M, Ohkusu K, Hata H, et al. Mycobacterium species identification - a new approach via dnaJ gene sequencing. Syst Appl Microbiol 2007; 30: 453-462.

44 Tirkkonen T, Nieminen T, Ali-Vehmas T, et al. Quantification of Mycobacterium avium subspecies in pig tissues by real-time quantitative PCR. Acta Vet Scand 2013; 55: 26

45 Matsuyama M, Ishii Y, Yageta Y, et al. Role of Th1/Th17 balance regulated by T-bet in a mouse model of Mycobacterium avium complex disease. J Immunol 2014; 192: 1707-1717.

46 Ito T, Connett JM, Kunkel SL, et al. The linkage of innate and adaptive immune response during granulomatous development. Front Immunol 2013; 4: 10.

47 Dickson RP, Erb-Downward JR, Freeman CM, et al. Bacterial topography of the healthy human lower respiratory tract. MBio 2017; 8: e02287-16. 